
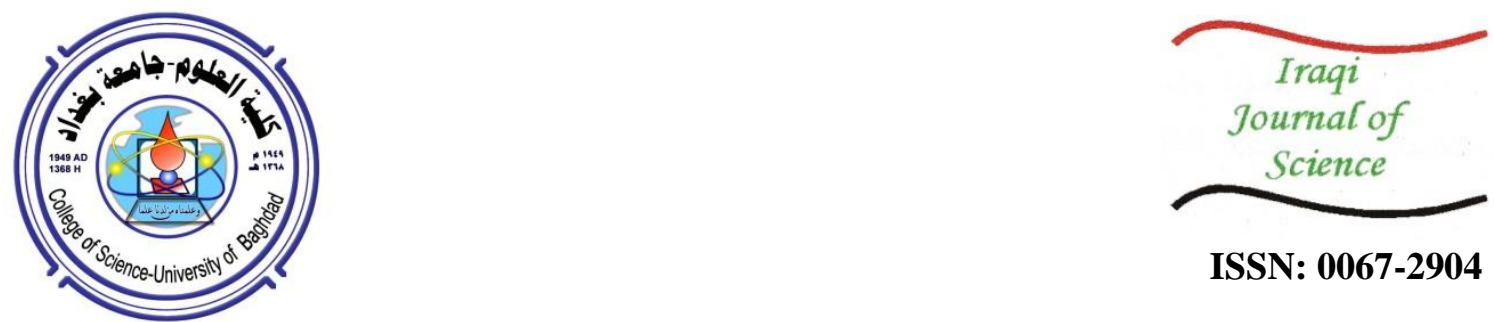

ISSN: 0067-2904

\title{
In Vitro Oncolytic activity of non-virulent Newcastle Disease Virus LaSota Strain against Mouse mammary adenocarcinoma
}

\author{
Sarah A.H. Hassan ${ }^{* 1}$, Aida B. Allawe ${ }^{1}$, Ahmed Majeed Al-Shammari \\ ${ }^{1}$ University of Baghdad, Department of Microbiology, College of Veterinary Medicine, Iraq \\ ${ }^{2}$ Mustansiriyah University, Iraqi Center for Cancer and Medical Genetics Research, Experimental Therapy \\ Department, Baghdad Iraq
}

Received: 23/7/ $2019 \quad$ Accepted: 28/8/2019

\begin{abstract}
Newcastle disease virus (NDV) is a wide-spectrum anti-tumor agent. The oncolytic selectivity of NDV, a family of Paramyxoviridae, depends on the differential type of inducing different death pathways. This work was conducted to further understand the oncolytic effect of LaSota strain. A mouse breast cancer model (Murine mammary adenocarcinoma cell line AMN3) was used in this study. Methyl Thiazolyl Tetrazolium (MTT) viability assay tested different NDV multiplicity of infection (MOI) values on mouse mammary adenocarcinoma cells incubated for 72 hours post-infection. The IC50 values and anti-tumor activity of LaSota strain against AMN3 cell line were determined. Following Hematoxylin and Eosin Stain, we examined the morphological modifications of IC50 along with 10 MOI values of NDV. The induction of NDV apoptosis in AMN3 cells was investigated using the technique of staining acridine orange and propidium iodide (AO / PI). Immunocytochemistry assay was performed using anti-NDV mAbs and caspases 8 and 9 to study NDV replication and apoptosis induction mechanisms. The lentogenic LaSota NDV strain, a live vaccine, demonstrated the oncolytic effect on mammary cancer cells of the AMN3 mouse and showed that LaSota strain triggered a dose-dependent increase in infected cells' apoptosis relative to untreated mammary cancer cells. The immunocytochemistry study showed that NDV infected cells were positive for virus infection and that caspase9 in mouse mammary cancer cells after LaSota strain infection was significantly enhanced compared to caspase 8 . In conclusions, NDV LaSota strain had oncolytic effects by destroying tumor cells and triggering the intrinsic apoptosis pathways in mouse mammary cancer cells. However, the mechanisms of the in vivo anti-tumor activity need to be better understood.
\end{abstract}

Keywords. NDV-Newcastle disease virus; Lentogenic NDV; Apoptosis; Breast cancer cells; Oncolytic therapy; MTT assay.

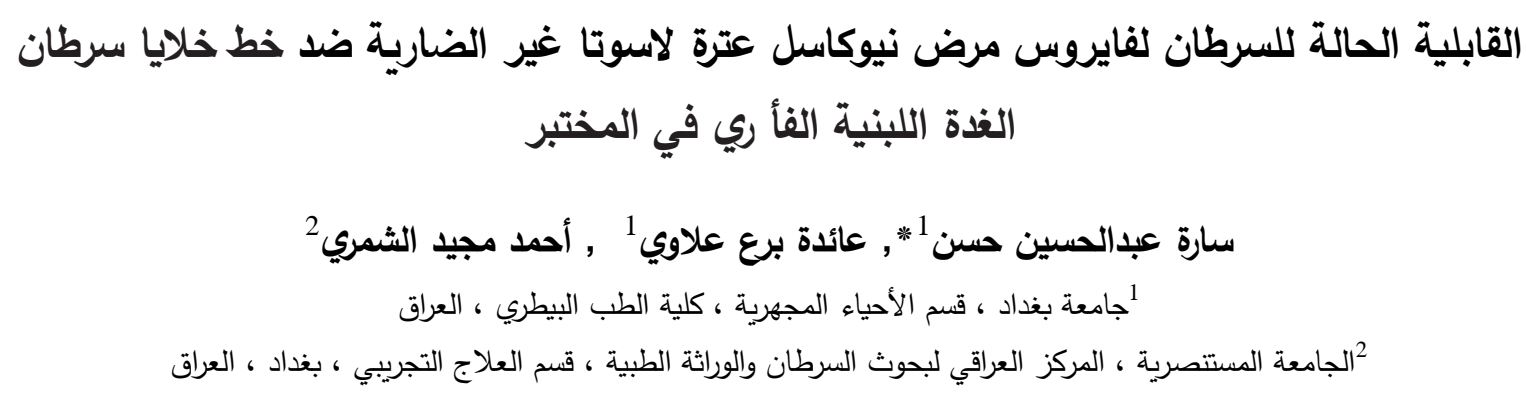




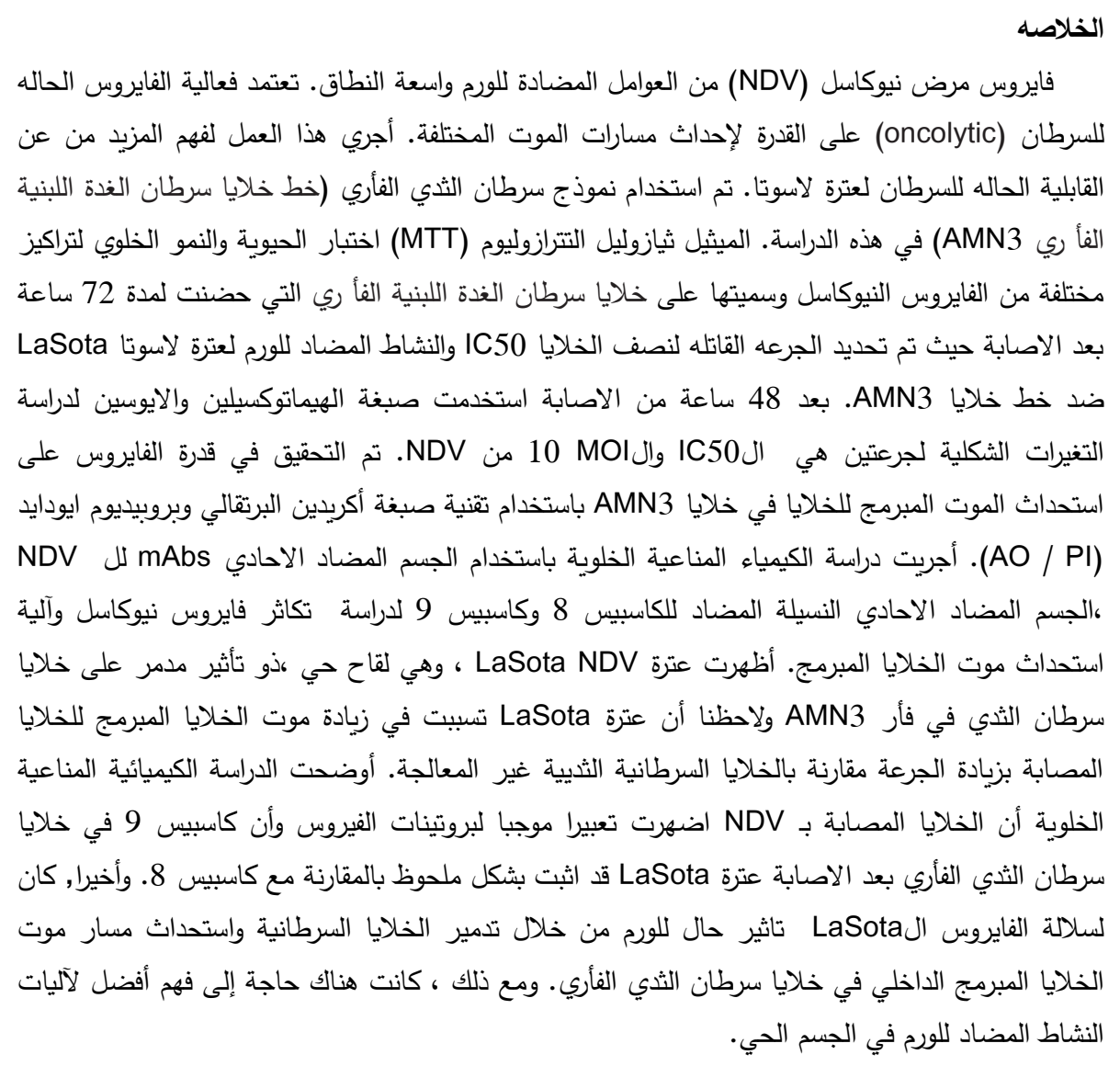

\section{Introduction}

Cancer is the leading cause of death globally [1]. Several factors are known to lead to the increased cancer incidence, such as environmental pollution [2]. Lately, virotherapy, which means using viruses as therapy for cancer, is recognized to have possible clinical applications for cancer treatment, as many viruses demonstrated anti-tumor properties with the power to suppress cancer cells without harming normal cells [3]. Newcastle disease virus (NDV) is a member of the new genus Avulavirus within the family Paramyxoviridae [4]. NDV is one of such oncolytic viruses which are presently being used in modern preclinical and clinical studies [5]. Csatary was the first to report arrested development of aggressive metastatic colon tumor in a Hungarian farm following an outbreak of NDV in 1971, indicating a correlativity with NDV infection [6]. Interest in the utilization of the anti-tumor NDV to kill tumor cells was due to its specifications in targeting tumor cells through three different mechanisms, cytolysis secondary to virus replication, apoptosis induction, and antigenic modification to the surfaces of tumor cells to make them more recognizable by the immune system [7]. It is also suggested that NDV may work as an anti-angiogenic agent [8]. Recently, it was found to suppress cancer cells through inhibition of the glycolysis pathway to enhance the anti-tumor activity [9]. Based on their pathogenicity in chickens NDV strains are classified into three types; velogenic, mesogenic, and lentogenic. The vaccination of poultry was satisfactory with the lentogenic or non-virulent attenuated strains such as NDV Hitchner-B1, Ulster, and LaSota, while the velogenic and mesogenic strains are considered as threats to agriculture. Therefore, lentogenic NDV strains have been employed widely as oncolytic viruses $[10,11,12]$. Moreover, the lentogenic strain LaSota was shown to enhance the conventional chemotherapies such as methotrexate and 5-Flurouracel [13, 14]. Rituximab and doxorubicin combination with NDV can work as anti-hematological cancer therapies [15]. Retinoic acid synergizes with attenuated NDV against digestive system tumors [16]. Bacterial hyaluronidase enhances attenuated the NDV potency in a mouse mammary tumor model [17].

Therefore, it is necessary to understand the mechanism behind virus replication in mouse tumor models and its efficiency as a model for human cancer treatment. The current study was designed to study the lentogenic LaSota NDV strain as an oncolytic agent against mouse mammary 
adenocarcinoma cells and to evaluate this activity through different parameters to facilitate further experiments that aim to develop NDV as an anti-tumor agent in preclinical experiments.

\section{Materials and Methods \\ Cell Line}

Murine mammary adenocarcinoma cell line AMN3 was grown in RPMI-1640 medium (US Biological, USA) supplied with $10 \%$ fetal bovine serum (FBS) (Capricorn Scientific, Germany), 100 units $/ \mathrm{ml}$ penicillin, and $100 \mu \mathrm{g} / \mathrm{ML}$ streptomycin and incubated at $37^{\circ} \mathrm{C}$. The AMN3 cell line was provided by the Cell Bank Unit, Experimental Therapy Department, Iraqi Center for Cancer and Medical Genetic Research (ICCMGR), Mustansiriyah University, Baghdad, Iraq. The AMN3 cell line was sub-cultured every three days, or when the cultures made $80 \%$ confluence. The cell line was tested regularly for mycoplasma contamination by the cell bank unit of the ICCMGR.

\section{LaSota strain}

LaSota strain, a lentogenic virulent strain of NDV used as lyophilized allantoic fluids (live vaccine), was obtained from Al Kindi for Production of Veterinary Vaccines and Drugs (IKLV) (Baghdad, Iraq). The virus was propagated in embryonated chicken eggs and tittered on Vero-slam cells for TCID50 determination and MOI calculation according to a standard procedure [18].

\section{Cytotoxicity MTT assay}

The MTT (Methyl Thiazolyl Tetrazolium) assay was carried on in order to assess the viability of the AMN3 cell line after infection with NDV LaSota strain. AMN3 cell line was seeded at 10,000 cells/well at a total volume of $100 \mu \mathrm{l}$ in growth medium. It reached a 70-80\% confluent monolayer. AMN3 cell line was infected with LaSota NDV for different multiplicity of infection (MOI) values $(0.1,0.5,1,3,5,10,15$ and 20$)$, whereas control wells remained untreated, aspiring only to the media and exchanging with free serum media. Viability of the cells was calculated after 72 hours of incubation. The old media from each well was removed, then $100 \mu \mathrm{l}$ of MTT solution were added into each well, followed by incubation for 2 hours at $37^{\circ} \mathrm{C}$ in the dark. The MTT solution was removedand $50 \mu 1$ of DMSO (Dimethyl Sulphoxide) (Santa Cruz Biotechnology, USA) were added to each well, followed by $37^{\circ} \mathrm{C}$ re-incubation for $30 \mathrm{~min}$. MTT was measured using an ELISA microplate reader (FLUOstar OPTIA Reader, Germany) and the absorbance was read at $584 \mathrm{~nm}$ wavelengths. Percentage of cytotoxicity was calculated as $(\mathrm{C}-\mathrm{T}) / \mathrm{C} * 100$, where $\mathrm{C}$ is the mean optical density of control wells, and $\mathrm{T}$ is the optical density of infected wells. The assay was performed in triplicate. Percent inhibition of tumor growth versus MOI graphs of the LaSota strain was obtained and the concentration causing half the maximum inhibitory (IC50) was determined [19].

\section{Cytopathological changes}

The AMN3 cell line was cultured on coverslips $(50,000$ cells/cover) at a total volume of $2 \mathrm{ml}$ in their serum culture medium. When the cells reached 70-80\% confluent monolayer in multi-well tissue culture plates (6-wells), they were exposed to the dose of IC50 and to $10 \mathrm{MOI}$ of LaSota strain in serum free media. The old culture media were removed and the cells were incubated for $2 \mathrm{~h}$ to allow the virus to infect the cells, then the media were aspirated and exchanged with new free serum media. The untreated control cells were prepared similarly without virus inoculum. Then the cells were incubated for $48 \mathrm{hrs}$. at $37^{\circ} \mathrm{C}$ with a moisturized atmosphere containing $5 \% \mathrm{CO}$. The infected cells were examined under an inverted light microscope to record the morphological alterations. After removing the suspension medium, the cells fixed with $10 \%$ formaldehyde were diluted in PBS for 10 min and stained with $\mathrm{H}$ and E stain. Finally, all slides were mounted with DPX and photographed by inverted microscope (Leica Microsystems, Germany) at 400x.

\section{Apoptosis assay}

The AMN3 cell line was cultured in a 96 well plate at a total volume of $100 \mu$ with a density of 10,000 cells/well in the growth medium until $70-80 \%$ confluent monolayer was formed. Cells were exposed to the virus dose of IC50 and to $10 \mathrm{MOI}$ values in serum free media for $2 \mathrm{hrs}$. After that the old media were exchanged with new serum free media. The AMN3 control was prepared similarly without virus inoculum. The cells were incubated for 48 hours at $37^{\circ} \mathrm{C}$ with $5 \% \mathrm{CO} 2$ and humidity. At the end point (48h), the medium was discarded, cells were stained using $1 \mu \mathrm{L}$ AO (acridine orange) and $1 \mu \mathrm{L}$ PI (propidium iodide) double staining at a ratio of 1:1 in $1 \mathrm{ml}$ of PBS at a total volume of $50 \mu \mathrm{l} /$ well for 20 seconds. Double staining was aspirated directly from the wells. The morphological changes of viable and apoptotic cells were observed and photographed by an inverted fluorescent microscope (Leica Microsystems, Germany). 


\section{Immunocytochemistry}

As described for cytopathological changes, the cells were cultured on coverslips and treated with the NDV dose of IC50 and 10 MOI values, whereas another group was left as control without virus infection. The cells were incubated at $37^{\circ} \mathrm{C}$ for 48 hours under a moisturized atmosphere with $5 \%$ $\mathrm{CO} 2$. Later, cells were fixed with cold acetone for $2 \mathrm{~min}$. Cells in $3 \% \mathrm{H} 2 \mathrm{O} 2$ and $1 \%$ fetal bovine albumin solution in PBS were blocked for 10 minutes. The cells were incubated with multiple primary antibodies (anti-NDV, anti-caspase8 and anti-caspase9diluted at 1:50 for 1-1.5 hours at room temperature. After washing with PBS, the cells were incubated with biotinylated mouse secondary antibody (diluted as 1:100) for 1 hour. After washing, the substrate DAB was prepared freshly in DAB buffer and sufficient drops of freshly prepared DAB substrate mixture were applied to cover the cell section. The slides were incubated in the dark for 20 minutes at room temperature. After that, the plates were washed with PBS and counterstained with hematoxylin stain for 5-10sec. They were then washed with distilled water and with PBS and immediately mounted using DPX on glass slides. The examination was performed using light microscopy.

\section{Quantitative image analysis}

The digital pictures of immunocytochemistry were used for quantitative analysis for the hematoxylin - DAB stained slides and taken with a Leica inverted microscope provided with a camera (Leica Microsystems, Germany). Three dissimilar staining zones of ICC images of each slide were analyzed in this study. Firstly, color, de-convolution assay was used for un-mixing with the DAB and hematoxylin stained areas, leaving a free image [20]. In addition, two other images were obtained; The first image is the hematoxylin stain and the second one is the DAB image, where the DAB image was quantified. The numerate of pixels of a specific intensity measure vs. their respective intensity was elevated utilizing "Fiji" version of ImageJ from 〈http: //Fiji. sc>[21]. We changed the intensity numbers in the data window to Optical Density (OD) numbers with the following formula: $\mathrm{OD}=\log$ (max intensity/Mean intensity), where max intensity $=255$ for 8 -bit images.

\section{Statistical analysis}

Data were shown as mean \pm SD for all measurements of triplicate observations. For MTT and ICC experiments, $\mathrm{n}=3$ images were used. One-way analysis of variance (ANOVA) multiple comparison was done to show variations among groups. The statistical significance analyses were done utilizing (GraphPad Prism version 7.0 for Windows, GraphPad Software, San Diego, CA, USA) to estimate the cytotoxicity and to detect the apoptotic impact of LaSota strain on AMN3 cell line in vitro. $\mathrm{p}<0.05$ was regarded as statistically significant.

\section{Results}

\section{In vitro anti-tumoral activity}

To determine the inhibitory effect of LaSota strain in vitro, we inoculated different $\mathrm{MOI}(0.1,0.5$, $1,3,5,10,15,20 \mathrm{MOI})$ of LaSota strain on monolayers of Murine mammary adenocarcinoma cell line AMN3. After 72 hours, the IC50 and tumor growth inhibition (GI) were determined (Table-1, Figure1). The anti-tumor behavior of LaSota strain showed that the increase in the dose of LaSota strain led to increasing the inhibitory effect of the virus on AMN3 cancer cells.

The cytotoxicity at the dose of 20 MOI LaSota strain was $57.5 \%$ with an estimated IC50 of 8.0 MOI after an exposure period of $72 \mathrm{hrs}$. 
A

AMN3 cell line 72 hour Cytotoxicity

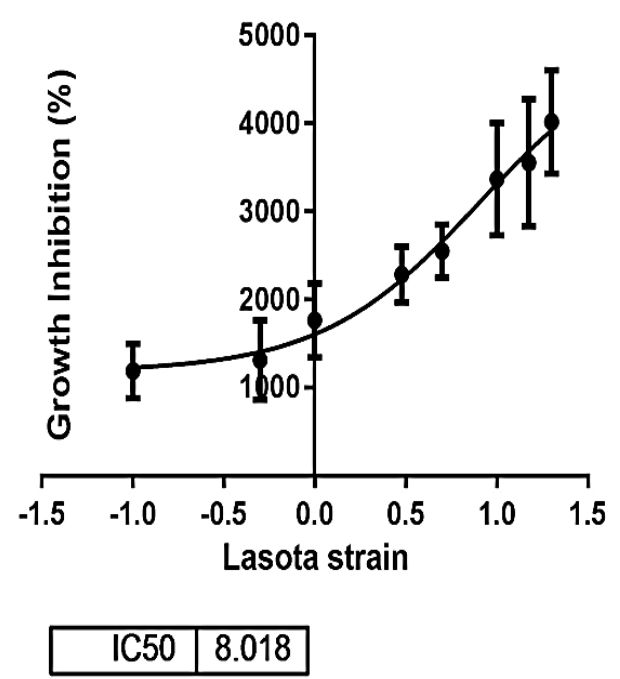

B

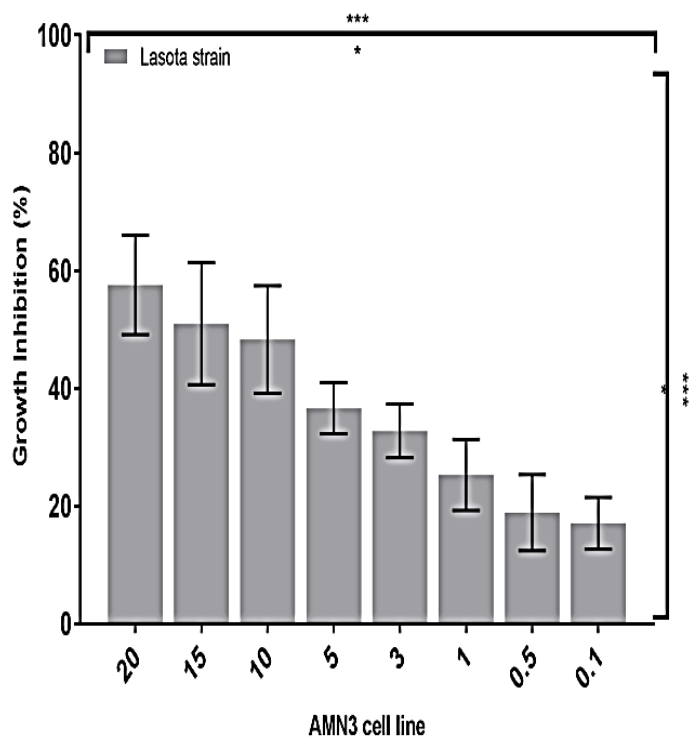

Figure 1- In vitro antitumor activity of LaSota strain. (A) IC50 of LaSota strain in AMN3 cell line (B) The AMN3 cell line (10,000 cells per well) were transferred in 96-well flat plates and LaSota strain $(0.1,0.5,1,3,5,10,15,20 \mathrm{MOI})$ was added in triplicates. After $72 \mathrm{~h}$, tumor growth inhibition was measured by the evaluation of MTT. For calculation of \% tumor growth inhibition (GI), the untreated cells were set to zero.

Table 1- The growth inhibition\% of LaSota strain on AMN3 cell line after 72 hours post exposure to ND virus.

\begin{tabular}{|c|c|}
\hline MOI of LaSota strain & Value of growth inhibition $(\mathbf{M} \pm$ SD) \% \\
\hline \hline $\mathbf{2 0}$ & $57.57 \pm 8.42$ \\
\hline $\mathbf{1 5}$ & $50.97 \pm 10.36$ \\
\hline $\mathbf{1 0}$ & $48.28 \pm 9.15$ \\
\hline $\mathbf{5}$ & $36.59 \pm 4.33$ \\
\hline $\mathbf{3}$ & $32.73 \pm 4.54$ \\
\hline $\mathbf{1}$ & $25.28 \pm 6.02$ \\
\hline $\mathbf{0 . 5}$ & $18.85 \pm 6.45$ \\
\hline $\mathbf{0 . 1}$ & $17.05 \pm 4.41$ \\
\hline \hline
\end{tabular}

\section{Cytopathology and detection of apoptosis}

The light inverted fluorescent microscope was utilized to observe the morphological alterations and the ratios of viable, necrotic and apoptotic cells in the population of AMN3 cell line exposed to the dose of $\mathrm{IC}_{50}$ and 10 MOI doses of NDV LaSota strain for 48h, compared to untreated AMN3 cell line. Under the light microscope, the unstained breast cancer cells were observed and showed round and shrinking cells. We also noticed an increase in cell granulation. In addition, the changes in the unstained culture showed large empty plaque spaces between cells (Figure-2 A2-A3) compared with the control cells that did not show any change for the same duration (Figure-2 A1). Whereas under the light microscope, the H\&E stained AMN3 cell line showed t many cytopathological changes which 
included vacuolation, cell degeneration, condensation of nucleus, and cells detachment (Figure-2 B2, B3), compared with the untreated cells Figure-(2B1). Furthermore, inverted fluorescent microscopy examination utilizing PI and AO was conducted; the selective permeability property of the entire plasma membrane of viable cells permitted the entry of AO stain, whereas they were impermeable to PI staining. The nuclei of viable cells were stained with green when observed under inverted fluorescence microscope, whereas the plasma membrane of necrotic cells was no longer intact which allowed PI to enter and make the cells appear red. The viable cells could be distinguished from apoptotic cells based on morphological deviations such as smaller cells size and fluoresced orange or red color. In addition, an increase in green to red shift was noticed in apoptotic LaSota strain-infected AMN3 cells (Figure-2C2-C3) after 48hrs, as compared with untreated control (viable green cell) (Figure-2 C1). This green to red shift in fluorescence was more at the treatment with the $10 \mathrm{MOI}$ doses than IC50 of LaSota strain; however, 10 MOI of LaSota strain induced more effect (apoptosis, compared to the $\mathrm{IC}_{50}$ dose.

contro1
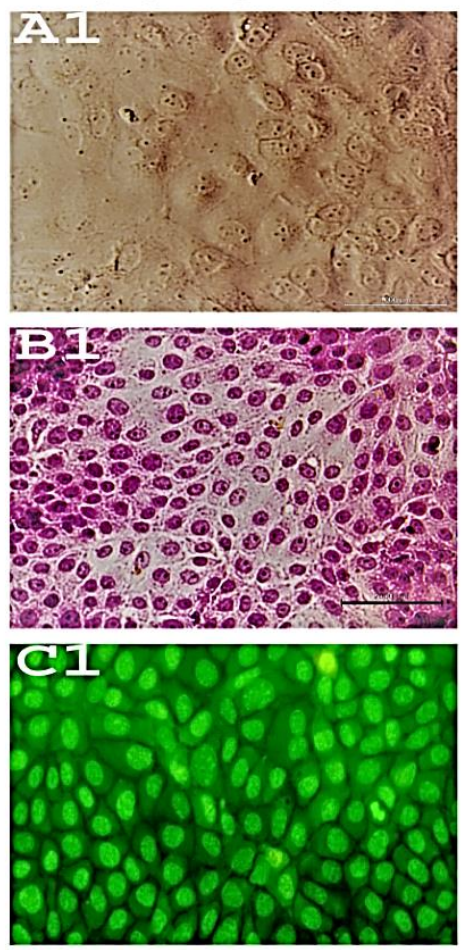

10 MOI
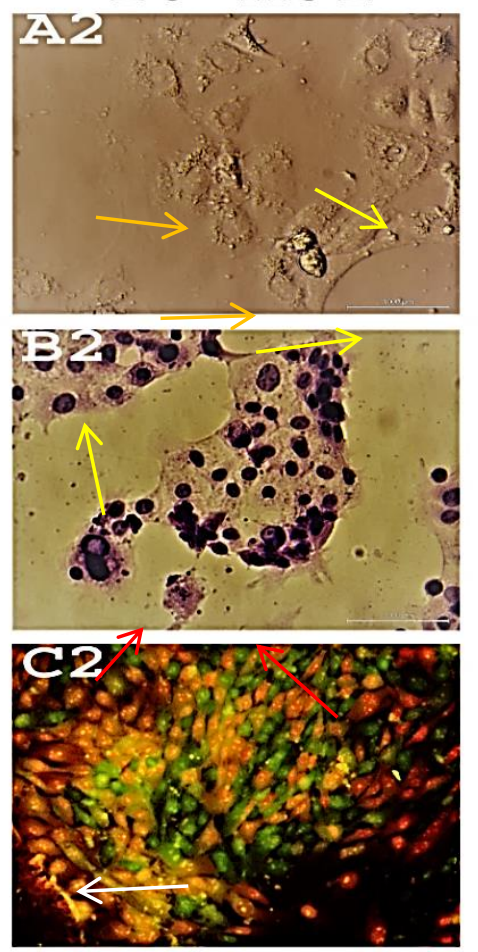

IC50
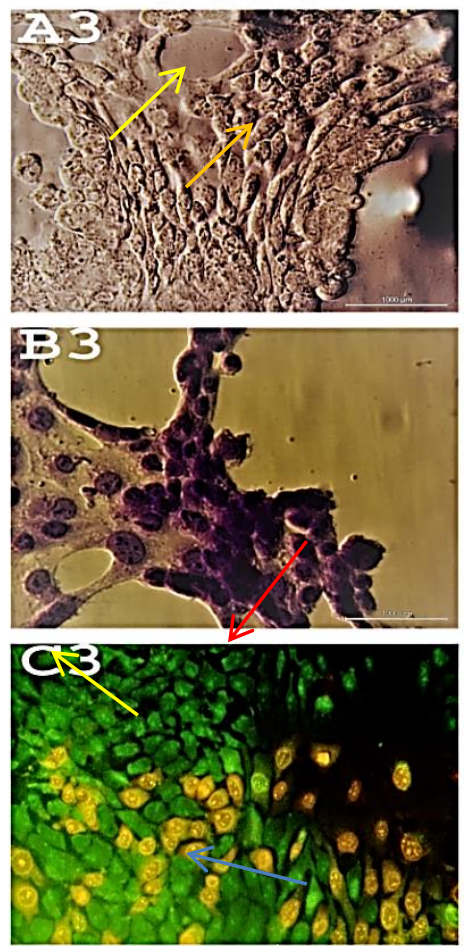

Figure 2-AMN3cell line treated for $48 \mathrm{hrs}$ with $\mathrm{IC}_{50}$ or10 MOI doses of LaSota strain afters; 400x. (A) In cross microscope, unstained cell showing the rounded cell (orange arrow) and vacuole disgeneration (yellow arrow). (B) In $\mathrm{H} \& \mathrm{E}$ staining, a treated celr showing vacuole degeneration (yellow arrow), hypertrophy, and condensation of nuclei (red arrow) compared to an untreated control cell. (C) In acridine orange and propidium iodide staining, a treated cell showed green, viable cells (blue arrow) and red apoptosis cells (white arrow) viewed under a fluorescence microscope.

\section{Detection of NDV and Caspase 8 and 9 proteins expression}

This assay was performed to identify the mechanism of apoptosis induction and determine the exact in vitro pathway for apoptosis of LaSota-treated AMN3 cell line after 48h post inoculation. Immunocytochemistry analysis showed that all tumor cells infected with LaSota strain were positive for the expression of ND and caspase 8 and 9 proteins Figures-(3A2-3, B2-3 and C2-3). Antigens in the infected cells were stained in brown, while no brown staining was observed in the positive control (uninfected cells) that were not exposed to LaSota strain but exposed to both primary and secondary antibodies Figures-(3A1, B1 and C1) (Nuclei were stained blue by counterstain).

\section{Digital Image Scoring}

Further analysis of ICC stained images $x$ revealed a significant $(\mathrm{P}<0.05)$ increase in optical density related to protein expression of anti-NDV and caspase9 in LaSota strain -Infected AMN3 breast cancer cell line as compared to the optical density of caspase 8 as well as to positive control, as judged 
by Digital Image Scoring using ImageJ program analysis of LaSota strain (10 MOI and IC50)-infected AMN3 cell line after $48 \mathrm{~h}$ post inoculation. Figure-3D shows demonstrative zones and pixel intensity analysis of ICC images. The analysis showed that the three proteins (anti-NDV, Caspase8, and Caspase9) were significantly expressed when compared to control and not stained cells (positive control), using ANOVA one-way multiple comparison test (Figure-3D1andD3). However, significant $(\mathrm{P}<0.05)$ increases in caspase9 activity and ND antibody in ND-infected AMN3 breast cancer cell were recorded as compared to caspase8 expression. Further, caspase8 show no significant difference $(\mathrm{P}<0.05)$ in optical density of expression after IC50 treatment Figure-3 D2, when compared with caspase9. These results indicated decreased caspase- 8 expression and, in contrast, increased caspase- 9 expression, which is considered as an interesting result reflecting that Caspase9 have an important effect in LaSota strain-stimulated cell death. Furthermore, the increase in the optical density was correlated with the dose of LaSota strain (Table-2).

Table 2-The optical densities of anti-NDV, Caspase8, and Caspase9 (M \pm SD) of IC50 and 10 MOI doses of LaSota strain on AMN3 cell line after 48 hours post-exposure

\begin{tabular}{|c|c|c|c|}
\hline \hline \multirow{2}{*}{ MOI of NDV } & \multicolumn{3}{|c|}{ The optical density of protein expression (M \pm SD) } \\
\cline { 2 - 4 } & Anti NDV & Caspase8 & Caspase9 \\
\hline \hline $\begin{array}{c}\text { LaSota strain } \\
\text { 10MOI }\end{array}$ & $0.556 \pm 0.038$ & $0.521 \pm 0.007$ & $0.563 \pm 0.029$ \\
\hline $\begin{array}{c}\text { LaSota strain } \\
\text { IC50 }\end{array}$ & $0.510 \pm 0.014$ & $0.499 \pm 0.059$ & $0.534 \pm 0.050$ \\
\hline Positive control & $0.426 \pm 0.022$ & $0.421 \pm 0.026$ & $0.427 \pm 0.024$ \\
\hline
\end{tabular}
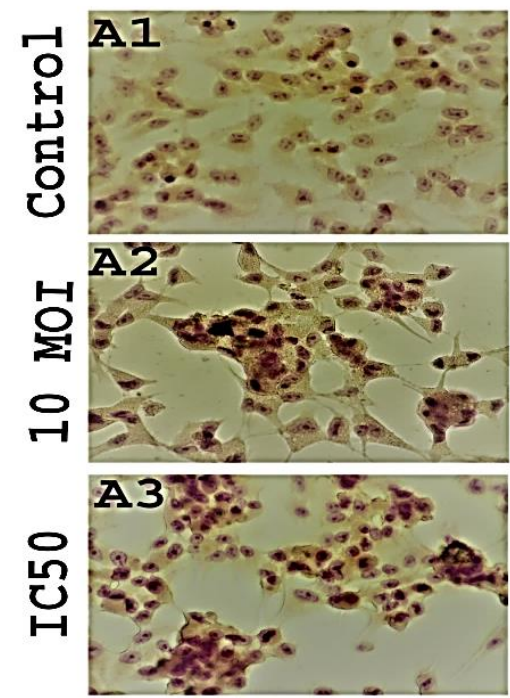

D1

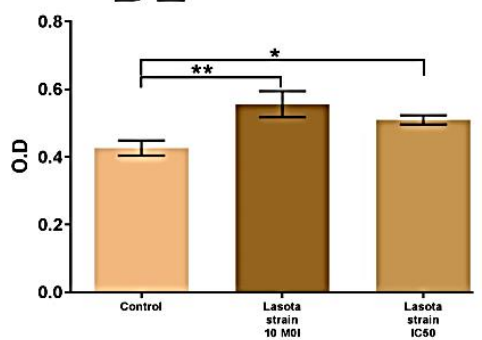

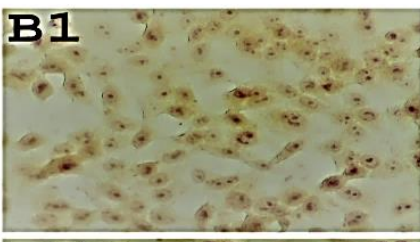
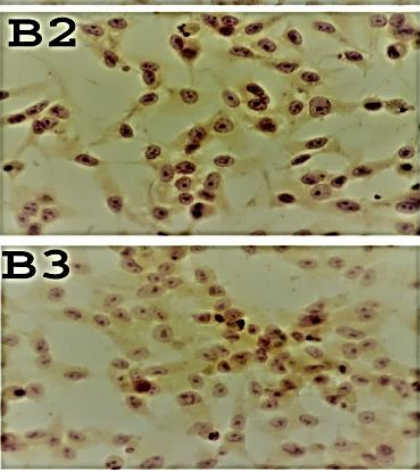

D2

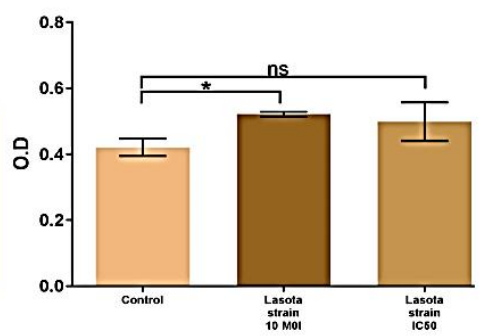

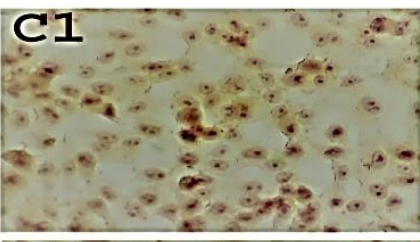
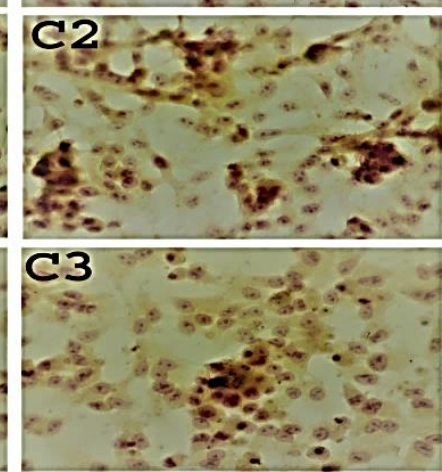

D3

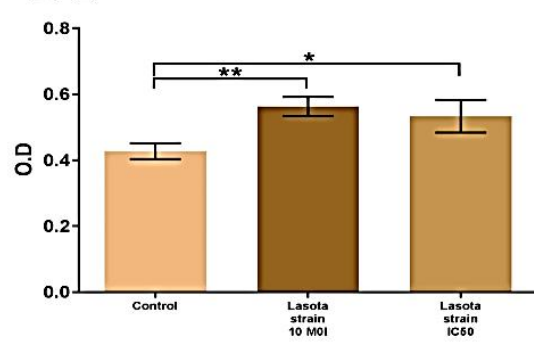

Figure 3- The results of immunocytochemistry on $\mathrm{AMN} 3$ cell line treated with $\mathrm{IC}_{50}$ and $10 \mathrm{MOI}$ doses of LaSota strain after $48 \mathrm{~h}$ of exposure to LaSota strain. Cancer cells take the brown cytoplasm stain (white arrow) (DAB stain 400x) compared to untreated control cancer cells which were negative to DAB stain (black arrow) (DAB stain 400x) using the primary antibody markers (A) ND antibody (B) caspase8 (C) caspas9 . (D) Digital Image Scoring showing significant proteins expression when stained with relative mAbs against the markers, as analyzed by ImageJ program. 
Data were analyzed by ANOVA one-way multiple comparison test. Differences were considered significant at $\mathrm{P} \leq 0.05$.

\section{Discussion}

In the current study, we used a lentogenic avirulent vaccine strain of NDV to evaluate oncolytic activities in our mouse model. The use of pathogenic replicative NDV strains, such as mesogenic or velogenic strains, for the treatment of cancer patients was reported to be problematic due to environmental biohazards associated with the pathogenicity of the virus and the potential viral spread. The attenuated NDV strains, such as HUJ, Ulster, and Hitchner-B1, selectively infect cancer cells and do not cause side effects in patients [22]. Besides, the NDV is a safe virus for the treatment of humans; its RNA genome is stable with the absence of genetic recombination, lack of antigenic drift, and lack of noticed human to human transmission [23].

The overall results from our study showed that NDV LaSota strain is effective against mouse mammary adenocarcinoma, in which the virus accomplishes its effect through cytolysis and apoptosis. In addition, the MTT assay was conducted to evaluate the effects of LaSota strains on cell viability of Murine mammary adenocarcinoma cell line (AMN3). The assay helped to find the inhibitory concentration (IC50) value of the virus that causes 50\% death of the tumor cells. The IC50 value obtained was 8.0MOI, while growth inhibition was $57.5 \%$ at $20 \mathrm{MOI}$ LaSota strain. These results manifest that the virus induced an efficient killing of 50\% of the AMN3, compared to untreated AMN3 cell, demonstrating its potent oncolytic effects. Elankumaran et al. [24] used 14 cell lines from a spectrum of malignancies which were infected with nonpathogenic NDV (rLaSota V.F) and rBCEdit viruses. The NDV showed a wider spectrum of cytotoxicity with a very low MOI. This could be due to the absence of expression of the $\mathrm{V}$ protein. The morphological alterations seen in the treated AMN3 cancer cells were confirmed by the cytopathological effects observed using $\mathrm{H}$ and $\mathrm{E}$ stain. Inverted microscopic analysis of the AMN3 cells treated with LaSota strain exhibitd round and shrinkage cells, which resulted in large, empty, plaque spaces between cells, while the untreated cells did not show any changes for the same duration. As shown by a previous study, HeLa cell-adapted NDV was used at passage number 5 and cytopathological impacts were identified by morphological changes which included cell shrinkage, rounding, detachment from the monolayer, and formation of giant cells (syncytium). Parental NDV (unadapted to HeLa cells)-infected HeLa cells appeared normal in gross morphology [25]. We further supported the effect of LaSota strain on tumor proliferation through induction of apoptosis and examining the PI/AO-stained cells under fluorescence microscopic. The results showed that LaSota strain increased the number of apoptotic cells in the infected AMN3 cancer cells in a dose-dependent manner. The viable cells could be distinguished from apoptotic cells based on morphological deviations such as the smaller cell size and the fluoresced orange or red, while the increase in green to red shift was noticed in LaSota strain-infected AMN3 cell line. On the other hand, the untreated AMN3 control cells demonstrated an increase in the viable green cells after 48 hours of treatment, demonstrating apoptosis features. This result proves the fact that the virus has an oncolytic effect on cancer cells. Al-Shamery et al. [26] revealedthat an Iraqi strain of NDV had in vitro effects on inducing apoptosis in AMN3, while the in vivo study found that the endoplasmic reticulum pathway is the dominant pathway for NDV in inducing apoptosis. The ability of the NDV to kill cancer cells has also been studied on many types of human cell lines, such as MDA-231and MCF7 , using six different vaccines of NDV strains [27]. These lentogenic strain have proven to be relatively effective in inhibiting tumor growth through apoptosis triggering.

Thus, the current study manifested that the LaSota strain of the virus possesses an in vitro anticancer activity on AMN3 cancer cells. The immunocytochemistry assay was conducted to detect protein expression. This assay was used to study the exact pathway of apoptosis by observing the expressed caspase 8 and 9 proteins and to examine the relativity of NDV infection by using anti-NDV $\mathrm{mAb}$ on infected AMN3 cancer cells after $48 \mathrm{~h}$ of LaSota strain inoculation. The results showed increases in the expression levels of the three protein in AMN3 cancer cells infected with LaSota strain, while non-treated cells were used for comparison. The results showed a decrease in the expression of caspase-8, as compared to an increase in caspase-9, which is an interesting result. This suggests that caspase-9 is necessary in mammary adenocarcinoma cells for NDV-induced apoptosis through mitochondrial pathways. Finally, the immunocytochemistry assay results were analyzed by an image $\mathbf{J}$ program; the results showed significant expression of all proteins when compared with LaSota strain non-treated cells, except that the IC50 did not affect caspas8 expression significantly $(\mathrm{P}<0.05)$. 
Further, caspase 8 showed less significant difference $(\mathrm{P}<0.05)$ in optical density of protein expression when compared with caspase9 . Our work is in agreement with the study of Humadai, T.J [28] who indicated a significant increase in the average percentage of cell expression of caspase 9 in the NDV exposed group, which implied that NDV had a powerful effect on inducing intrinsic (mitochondrial) apoptotic pathways [29]. Furthermore, our findings are in agreement with those of a previous study on cells of mouse fibroblasts (3T3) and human colon adenocarcinoma (HT-29) cells [30].

Finally, we indicate that the LaSota strain showed a remarkable antitumor activity against AMN3 mouse mammary adenocarcinoma cells, which was induced through cytolysis and apoptosis via intrinsic (mitochondrial) pathway. This effect was more dictated by the increased percentage of caspase-9 expression as compared to that of caspase8.

\section{Acknowledgments}

The authors would like to extend their thanks to the Department of Experimental Therapeutics, Iraqi Center for Cancer and Medical Genetic Research, Mustansiriyah University, Baghdad, Iraq. We also like to thank the specialist Dr. Aida Bara Allawe, for valuable guidance during research work and Dr. Ahmed M. Al-Shammari for the use of their laboratory facilities.

\section{References}

1. Siegel, R.L., Miller, K.D. and Jemal, A. 2019. Cancer statistics. CA: A Cancer Journal for Clinicians, 2019. 69(1): 7-34.

2. Al-Shammari, A.M. 2016. Environmental pollutions associated to conflicts in Iraq and related health problems, in Reviews on Environmental Health. p. 245.

3. You, L. 2004. Future directions: oncolytic viruses. Clinical Lung Cancer. 5(4): 226-230.

4. Alexander, D. 1988. Newcastle disease virus-An avian paramyxovirus, in Newcastle disease. Springer. p. 11-22.

5. Schirrmacher, V. 2016. Fifty Years of Clinical Application of Newcastle Disease Virus: Time to Celebrate! Biomedicines. 4(3): 16.

6. Csatary, L. 1971. Viruses in the treatment of cancer. The Lancet. 298(7728): 825.

7. Al-Shammary, A.M., H.H. Hassani, and U.A. Ibrahim. 2014. Newcastle disease virus (NDV) Iraqi strain AD2141 induces DNA damage and FasL in cancer cell lines. Journal of Biology and Life Sciences. 5(1): 1.

8. Al-Shammari, A.M., Z.A. Al-Hili, and Yaseen, N.Y. 2013. 647. Iraqi Newcastle Disease Virus Virulent Strain as Cancer Antiangiogenic Agent. Molecular Therapy. 21: 1.

9. Al-Shammari, A.M. 2016. 2-Deoxyglucose and Newcastle Disease Virus synergize to Kill Breast Cancer Cells by Inhibition of Glycolysis Pathway Through Glyceraldehyde3-Phosphate Downregulation.

10. Dortmans, J. 2010. The viral replication complex is associated with the virulence of Newcastle disease virus. Journal of virology. 84(19): 10113-10120.

11. Tayeb, S., Zakay-Rones, Z. and. Panet, A. 2015. Therapeutic potential of oncolytic Newcastle disease virus: a critical review. Oncolytic virotherapy. 4: 49.

12. Alkayyal, A.A., A.B. Mahmoud, and Auer, R.C. 2016. Interleukin-12-expressing oncolytic virus: A promising strategy for cancer immunotherapy. Journal of Taibah University Medical Sciences. 11(3): 187-193.

13. Al-Shammari, A.M. 2016. In vitro synergistic enhancement of Newcastle Disease Virus to 5fluorouracil cytotoxicity against tumor cells. Biomedicines. 4(1): 3.

14. Al-Shammari, A. and Yaseen, N. 2012. In Vitro Synergistic enhancement of Newcastle Disease Virus to Methotrexate cytotoxicity against tumor cells. Al-Anbar J. Vet. Sci. 5:. 102-109.

15. Al-Shammari, A.M., Rameez, H. and Al-Taee, M.F. 2016. Newcastle disease virus, rituximab, and doxorubicin combination as anti-hematological malignancy therapy. Oncolytic virotherapy. 5: 27.

16. Al-Shammari, A.M. 2019. Enhancement of Oncolytic Activity of Newcastle Disease virus Through Combination with Retinoic Acid Against Digestive System Malignancies. in Molecular Therapy. Cell Press 50 Hampshire St, Floor 5, Cambridge, Ma 02139 USA.

17. Salih, H.S., Al-Shammari, A.M. and Laftaah, B.A. 2018. Intratumoral Co-Administration of Oncolytic Newcastle Disease Virus and Bacterial Hyaluronidase Enhances Virus Potency in Tumor Models. Journal of Global Pharma Technology. 10(10): 303 - 310. 
18. Salih, R.H. 2017. Antiviral effects of olea europaea leaves extract and interferon-beta on gene expression of newcastle disease virus. Adv. Anim. Vet. Sci. 5(11): 436-445.

19. Takimoto, C.H. 2003. Anticancer drug development at the US National Cancer Institute. Cancer chemotherapy and pharmacology. 52(1): 29-33.

20. Chatterjee, S. 2013. Quantitative immunohistochemical analysis reveals association between sodium iodide symporter and estrogen receptor expression in breast cancer. PloS one. 8(1): e54055.

21. Schindelin, J. 2012. Fiji: an open-source platform for biological-image analysis. Nature methods. 9(7): 676.

22. Freeman, A.I. 2006. Phase I/II trial of intravenous NDV-HUJ oncolytic virus in recurrent glioblastoma multiforme. Molecular therapy, 13(1): 221-228.

23. Mayo, M. 2002. A summary of taxonomic changes recently approved by ICTV. Archives of virology. 147(8): 1655-1656.

24. 24. Elankumaran, S., Rockemann, D. and Samal, S.K. 2006. Newcastle disease virus exerts oncolysis by both intrinsic and extrinsic caspase-dependent pathways of cell death. Journal of virology. 80(15): $7522-7534$.

25. Vigil, A. 2007. Use of reverse genetics to enhance the oncolytic properties of Newcastle disease virus. Cancer research. 67(17): 8285-8292.

26. Alwan, M.J., Yaseen, N.Y. and Al-Shamery, A.M. 2011. Study the apoptotic pathway induced in mammary adenocarcinoma tissue treated by local Iraqi strain of Newcastle disease virus. Iraqi Journal of Cancer and Medical Genetics. 4(1): 40-47.

27. Freshney, R.I. 2015. Culture of animal cells: a manual of basic technique and specialized applications: John Wiley \& Sons.

28. Humadai, T.J. 2007. Apoptotic Induction Mechanism in Tumor Cells Treated by Newcastle Disease Virus in vitro and in vivo, in Veterinary Medicine-Pathology. Baghdad/ iraq: Veterinary Medicine College.

29. Al-Shammari, A.M. 2015. Oncolytic Newcastle Disease Virus Iraqi Virulent Strain Induce Apoptosis In Vitro through intrinsic Pathway and Association of Both Intrinsic and Extrinsic Pathways In Vivo. Molecular Therapy. 23: S173-S174.

30. Assayaghi, R.M., Alabsi, A.M. and Ali, A.M. 2016. Apoptosis induction of newcastle disease virus strains (AF 2240 \& V4-UPM) on HT-29Human colorectal adenocarcinoma cells. $J$ Cancer Res. 4: 101. 